\title{
CHANGEOVER PROCESS ASSESSMENT OF WARP-KNITTING LET-OFF EQUIPPED WITH MULTISPEED ELECTRONIC LET-OFF SYSTEM
}

\author{
Yong $\mathrm{Ji}^{1,2, \star *}$, Gaoming Jiang ${ }^{1,3, *}$, Mengting Tang ${ }^{1,3}$ \\ 1 Engineering Research Center for Knitting Technology, Ministry of Education, Jiangnan University, Jiangsu, Wuxi, 214122, China \\ 2 School of Textile and Clothing, Nantong University, Jiangsu, Nantong 226019, China \\ 3 International Joint Research Laboratory for Noval Knitting Structural Materials, Jiangnan University, Jiangsu, Wuxi, 214122, China \\ *Corresponding author. E-mail: jgm@jiangnan.edu.cn, europelinkage@163.com
}

\begin{abstract}
:
Warp-knitted fabrics have gained increasing attention due to their excellent properties, and their production is strongly dependent on the warp-based electronic let-off system. Most of the existing control systems are single speed electron let-off structure, which brings a series of issues such as broken yarn, missed needle, and running thread caused by let-off delay, making it hard for manufacturer to produce the required fabrics. To fill the gap, this article proposes a new framework of a multispeed electronic let-off system for warp knitting based on closedloop control strategy. As a demonstration of the capacity and generalizability of this newly proposed methodology, the 01-type four-channel acoustic vibration analyzer is used to test and analyze the time of switching, the let-off amounts, and the change in warp tension during the process of switching let-off amounts, including the explanation and comparison of difference values of let-off quantities. It is shown that the proposed method for the production of warp-knitted fabrics is a useful method for achieving digital manufacture of warp-knitted fabrics.
\end{abstract}

\section{Keywords:}

Warp knitting, multispeed let-off, let-off amounts, warp tension

\section{Introduction}

The rapid development in knitting has boosted warp- and weft-knitting industries. With the increasing achievements in automation technologies and the improvement in people's aesthetics, there is continuously growing demand for highquality patterns and appearances in high-end warp-knitting technology, which has further improved the automation technologies in warp-knitting industry [1]. Currently, high-speed warp-knitting machines are able to weave all kinds of complex patterns, making it possible to apply multispeed electronic letoff systems in a wide range [2]. However, multispeed electronic let-off systems have higher requirements than single-speed electronic let-off systems (e.g., in terms of let-off accuracy, responsiveness, and stability). Especially, in multispeed electronic let-off systems, the switching of let-off amounts should be completed in a short time to ensure the stability of the let-off system; otherwise, problems such as yarn breakage and needle leakage, affecting the cloth's surface, and even the phenomenon of warp smashing, affecting the normal warp knitting, are easy to occur [3].

Numerical simulation methods were widely applied to investigate warp-knitted fabrics properties. For instance, $\mathrm{Hu}$ and Jiang [4] used a model to predict the surface formability of a multiaxial warp-knitted fabric, which shows that the diagonal yarns tend to gather along the weft direction of the fabric by modeling of the forming behavior of multiaxial warp-knitted fabrics. A yarn numerical model proposed by Jiang and Chen [5] was employed to present geometric and algebraic algorithms and relevant optimizing operators of yarns in fabric, which defines more realistic and flexible geometry models based on differential structure of yarns. In addition, Wang and Hu [6] confirmed that the structural parameters of traction density, linear density, and types of yarn have strong influence on the let-off amounts of warp-knitted fabric. Meantime, the surface deformation of the three-dimensional (3D) warp interlock fabric has been analyzed by Dufour et al. [7]. They pointed out that based on the different slope of the structure and the punch press, the yarn strain has good correlation with the local strain measured by the sensor. Zhang et al. [8] investigated the relationship between warp-knitted run-in values and process parameters which involves yarn fineness, overlapping, and underlapping. Based on the response surface methodology analysis, there is a certain influence of process parameters on warp-knitted run-in values. In addition, more precise numerical models have been reported by other researchers.

Warp-knitting technology has been upgraded from simple mechanical let-off system to electronic let-off system. However, the existing research considers only the change of let-off amounts in single-speed electronic let-off system from the perspectives of control structure and control algorithm. In comparison, there is rare research on the influence of the varied let-off amounts switching on the response characteristics of multispeed let-off system. As we know that it is important to evaluate the influence of let-off volume between sequences under the condition of high-speed let-off system. The aim of this study was to establish a warp-knitting multispeed electronic let- 
off system based on closed-loop control and to experimentally investigate the response characteristics and warp tension of the designed multispeed electronic let-off system during the process of switching let-off amounts. The main influencing factors of the system are multispeed warp feeding, switching of let-off quantity, and adjustment of warp tension. Therefore, the response characteristics of multispeed let-off system with closed-loop control and the change rule of warp tension are analyzed.

\section{Methodology}

In the experimental design, different let-off amounts (200, 400, 800 , and $1,200 \mathrm{~mm} / \mathrm{rack}$ ) are set to study the switching time and warp tension. A four-channel acoustic vibration analyzer is used to collect the time-speed curves and the warp tension curve during the switching process [9]. First, the two speed interfaces of the analyzer are used to acquire the spindle speed signal and the motor speed signal. The real-time spindle speed and motor speed are obtained through the analyzer's software. The real-time data of the motor speed are calculated to obtain the switching time under the conditions of different let-off speeds and let-off amounts [10]. Then the signal of warp tension sensor is transmitted to the acceleration interface of the analyzer, thereby acquiring the relationship between the warp tension and the motor speed [11]. Thus, we can identify the curve between the warp tension and the time. Then analyzer is used for angle domain analysis to obtain the warp tension curve with the loop angle. Finally, we can analyze how motor speed influences the warp tension [12].

The experiment is conducted on an experimental test platform. The warp-knitting machine is Karl Mayer HKS4 with a highspeed let-off system based on closed-loop control. Then, the warp beam GB3 has $900 \mathrm{~mm}$ outer circumference, $630 \mathrm{~mm}$ inner circumference, 7,820 remaining laps, and $251.2 \mathrm{~mm}$ outer circumference of press roller. The warp beam GB4 has $1,130 \mathrm{~mm}$ outer circumference, $630 \mathrm{~mm}$ inner circumference, and 9,900 remaining laps. To consider the change of varied let-off amounts, four sets of comparative experiments were designed at speed of 1,300 rev/min as summarized in Table 1.

The switching time between multiple let-off amounts is accurately measured in this experiment using LMS test. Lab software, the speed signal of the spindle motor and warp motor, and tension sensor signal are transmitted to the two speed interfaces and the acceleration interfaces [13]. The sampling period and sampling frequency are appropriately selected to accurately determine the switching time between varied let-off amounts [14]. Under the condition of $500 \mathrm{~ms}$ sampling period and $1 \mathrm{kHz}$ sampling frequency, we obtain the speed curve of the spindle motor and acquire the warp tension curves with varied let-off amounts switching.

\section{Results and discussion}

\subsection{A closed-loop control model of the warp-knitting multispeed let-off}

The designed multispeed electronic let-off model mainly consists of the following four parts: the let-off control unit, the let-off drive device, the warp beam speed feedback device, and the spindle speed capture device as shown in Figure 1. A new type of DSP is used as the main control unit, an AC servo motor as the drive device, and a speed measuring roller as the warp beam speed feedback device. The speed of the warp beam is captured in real time to obtain the required speed of the next cycle of the motor. Besides, the fuzzy PID control algorithm is

Table 1. Technological parameter of knitting process

\begin{tabular}{|c|c|c|c|}
\hline $\begin{array}{c}\text { Experiment } \\
\text { No. }\end{array}$ & GB3 & GB4 & $\begin{array}{c}\text { Let-off quantity } \\
\text { difference }(\mathrm{mm} / \\
\text { track })\end{array}$ \\
\hline 1 & $(1-0 / 0-1)^{*}(1,040 \mathrm{~mm} / \mathrm{rack}) /(1-0 / 1-2)^{*}(1,240 \mathrm{~mm} / \mathrm{rack})$ & $(1-0 / 1-2)^{*}(1,200 \mathrm{~mm} / \mathrm{rack})$ & 200 \\
\hline 2 & $(1-0 / 1-2)^{*}(1,240 \mathrm{~mm} / \mathrm{rack}) /(1-0 / 2-3)^{*}(1,640 \mathrm{~mm} / \mathrm{rack})$ & $(1-0 / 1-2)^{*}(1,200 \mathrm{~mm} / \mathrm{rack})$ & 400 \\
\hline 3 & $(1-0 / 1-2)^{*}(1,240 \mathrm{~mm} / \mathrm{rack}) /(1-0 / 3-4)^{*}(2,040 \mathrm{~mm} / \mathrm{rack})$ & $(1-0 / 1-2)^{*}(1,200 \mathrm{~mm} / \mathrm{rack})$ & 800 \\
\hline 4 & $(1-0 / 1-2)^{*}(1,240 \mathrm{~mm} / \mathrm{rack}) /(1-0 / 4-5)^{*}(2,440 \mathrm{~mm} / \mathrm{rack})$ & $(1-0 / 1-2)^{*}(1,200 \mathrm{~mm} / \mathrm{rack})$ & 1,200 \\
\hline
\end{tabular}

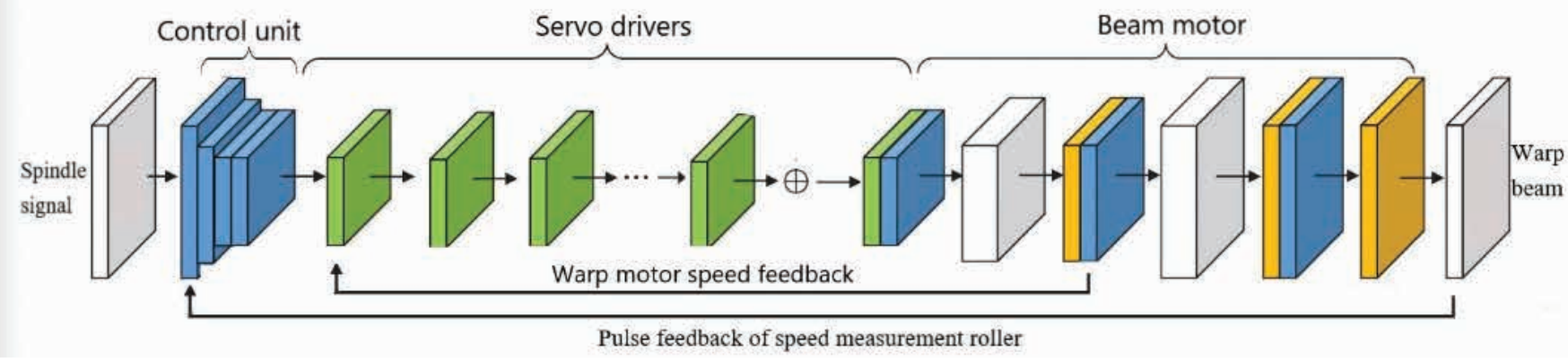

Figure 1. Control flow of warp-knitting multispeed let-off model. 
used to correct the motor speed in real-time manner to ensure the efficiency and accuracy.

\subsection{The influencing factors of varied let-off amounts switching}

In the actual warp-knitting production, the main control unit calculates the let-off amounts of the current sequence according to the control algorithm, followed by calculating the required speed. Then, the motor is powered by the servo drive to run at the required speed [15]. As the command of let-off amounts changes, the system should fulfill the duty to complete the switching of the let-off amounts. Then, the control unit calculates the speed corresponding to the let-off amounts [16]. Later, the motor is powered by the servo drive to the required speed, thereby completing the switching of the let-off amounts.

The response characteristics of the let-off system describe the behaviors under the condition of a sudden change in the motor speed, which can be intuitively expressed by the time to complete the switching between varied let-off amounts. The change of warp tension can also indirectly reflect the response characteristics of the let-off system [17]. In the normal warpknitting process, there is a periodic change in the warp tension; when the warp-knitting machine weaves, the warp let-off can be regarded as a movement with constant speed, but in the process of warp winding a synthetic ring must be equipped to complete the looping action such as needle traverse, needle back traverse, etc. Therefore, the required yarn amounts vary with different cycles, formulating a periodic fluctuation in the warp tension. In the normal warp-knitting process, the warp tension does not show large fluctuations [18]. However, the influence factors of varied let-off amounts switching should be considered for multispeed let-off system, because a sudden change in the let-off amounts will greatly fluctuate the motor speed. Besides, there is a delay resulting from the response time and mechanical transmission interval in the electronic letoff system, making the motor speed cannot reach the required speed in a timely manner, resulting in the difference in the required yarn amounts and the actual amounts. This indirectly causes fluctuations in warp tension and the instability of the entire system, further leading to the problems such as running yarn and yarn breakage [19]. Therefore, for the multispeed let-off system, the accuracy and stability are significantly influenced by the switching time and the warp tension.

\subsection{The control principle of feed quantity switching between sequences}

In the process of switching the let-off amounts, motor speed is closely related to the difference in the let-off amounts. Greater difference of $\Delta L$ leads to the larger fluctuation in motor speed $\Delta W_{\mathrm{b}}$, therefore it increases the switching time $\Delta T$ :

$$
\Delta T=\frac{\Delta W_{\mathrm{b}}}{k_{1} A}
$$

where $\Delta T$ represents the time required to complete the speed switching, $\Delta W_{\mathrm{b}}$ represents the difference in the let-off amounts, $A$ represents the acceleration of the motor, and $k_{1}$ represents the time conversion. In actual production, when the machine is running at a certain speed, the relationship between the let-off amounts and the motor speed is expressed as:

$W_{\mathrm{b}_{\mathrm{i}}}=\frac{W_{0} M F_{0}}{k_{2} B}$

According to the relationship between the let-off amounts and motor speed, we can obtain the values of $\Delta F$ and $\Delta W_{\mathrm{b}}$, so that the theoretical switching time is obtained:

$\Delta W_{\mathrm{b}}=\frac{W_{0} M \Delta F}{k_{2} B}$

$T=\frac{W_{0} M \Delta F}{k_{3} A B}$

where $W_{0}$ represents the current spindle speed of the machine, $B$ represents the current outer circumference of the warp beam's head, $k_{2}$ and $k_{3}$ represent the time conversion, and $M$ is the reduction ratio of the speed reducer. It can be seen from the equations that the switching time is mainly proportional to the difference between let-off amounts and the spindle speed. However, the switching time is inversely proportional to two parameters: one is the current outer circumference of the warp beam's head and the other is the acceleration of the motor.

The acceleration $A$ of the motor is related to its speed characteristic [20]. The acceleration $A$ of the servo motor is not a fixed value, and it has a close relationship with the actual speed and target speed fed back by the motor [21]. When $\Delta W_{\mathrm{b}}$ becomes large, the servo system will have a gain adjustment, and $A$ will become larger, so the switching time is a very complicated variable [22]. To study the switching time between different let-off amounts, a four-channel acoustic vibration analyzer is used to collect the time-speed curves and the real-time dynamic curves of the warp tension during the switching process. Then, comprehensive analysis is performed on the above two curves.

\subsection{Analysis of the switching time between varied let-off amounts}

Using the four-channel acoustic vibration analyzer, the timespeed curve (Figure 2) is obtained when switching the let-off amounts. Under the condition of constant machine speed, there is a fluctuation in the curve, obvious rising and falling during the normal warp-knitting process, change in the let-off motor speed due to change in the let-off amounts. As the letoff amounts decrease the let-off motor will decelerate to the required speed, and as the let-off amounts increase, the letoff motor will accelerate to the required speed. Moreover, with the increase in the let-off amounts difference, the let-off motor speed is continuously increased when the let-off amounts are low. 


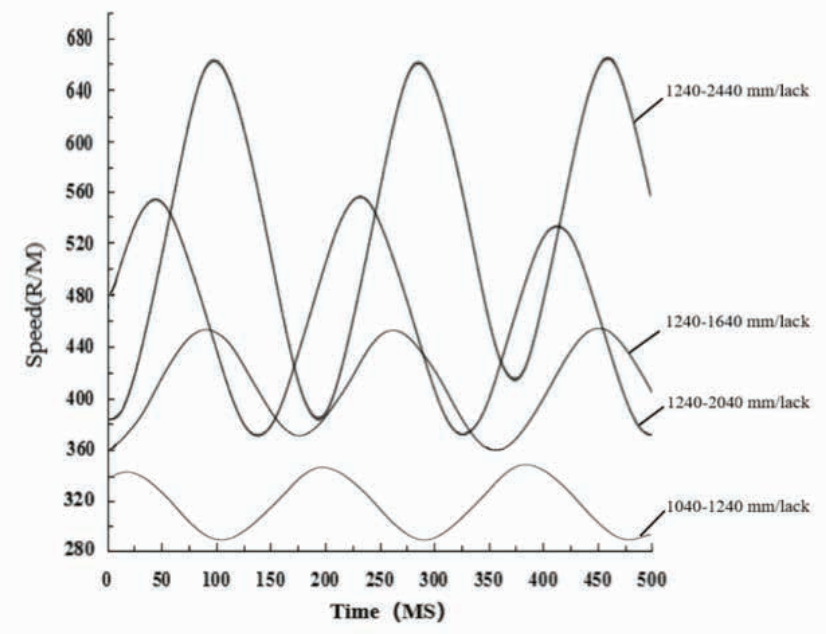

Figure 2. The speed change curve of beam motor with varied let-off amounts switching.

Under the condition of constant spindle speed, the relationship between the let-off amounts and the let-off motor speed is shown in Eq. (2). According to Eq. (2), the theoretical speed of the let-off motor is $1,300 \mathrm{rev} / \mathrm{min}$. According to the time-speed curve acquired by the analyzer at the speed of $1,300 \mathrm{rev} / \mathrm{min}$, we can obtain the actual speed of the let-off motor and the corresponding switching time under the condition of different let-off amounts. Table 2 presents the switching time required by the let-off motor when switching the let-off amounts.

\subsection{Warp tension change with switching let-off amounts}

To study the variation rule of warp tension with switching letoff amounts, the difference values of let-off amounts were collected as $200,400,800$, and $1,200 \mathrm{~mm} /$ rack. Figure 3 shows the fluctuation curve of warp tension changing with the loop-forming angle and switching of different groups of let-off amounts sequence. It can be seen that the warp tension is not a fixed value during the knitting process, but it has periodic fluctuation, especially large fluctuation with switching the letoff amounts. When switching from the low let-off sequence to the high let-off sequence, the let-off system has lag caused by intermittent mechanical transmission. The motor cannot reach the target speed in a short time so as to tighten the yarn and fluctuate highly in tension. Therefore, maintaining stable average tension and peak tension in the warp-knitting process is the prerequisite to ensure the normal running of machines.

To study the change of warp tension during the switching process, according to the fluctuation curve and related data of warp tension changing with the winding angle when warpknitting sequence switching with different let-off amounts. We collected the average value and peak value of warp tension when the let-off amounts of each tissue sequence were switched as given in Table 3.

It can be seen that the warp tension under low let-off amounts shows lower average and peak values than those under high let-off amounts. It indirectly verifies that the actual speed of the motor is larger than the theoretical speed under low let-off amounts so as to loose yarn and reduced tension. Therefore, small average and peak warp tension are observed under low let-off amounts. When the let-off amounts increases from low level to high level, there is an increase in both the average and peak values of the warp tension, as a result of the essential characteristics of the motor; accordingly, the yarn becomes tight and the tension fluctuation rapidly increases in a very short manner. Therefore, the average and peak values of the warp tension are larger than the normal values. Through the angle domain analysis, the peak angle of yarn tension is $196^{\circ}$ in the loop formation. According to the change of the peak angle of tension in the loop formation, the tension fluctuation of the warp is larger than that of the normal warp-knitting sequence when the let-off sequence is switched.

\section{Conclusion}

A closed-loop control model of the warp-knitting multispeed let-off was presented in this article. The developed model considered the effect of various let-off amounts in warp-knitting process. The effect of the difference in the let-off amounts on the switching time and warp tension is analyzed. By capturing the real-time speed curves of the motor during the switching between different let-off amounts, there is a certain deviation

Table 2. Switching time of varied let-off amounts

\begin{tabular}{|c|c|c|c|c|c|}
\hline $\begin{array}{l}\text { Let-off amounts } \\
\text { (mm/lack) }\end{array}$ & $\begin{array}{c}\text { Difference of } \\
\text { let-off amounts } \\
\text { (mm/lack) }\end{array}$ & Switching time & $\begin{array}{c}\text { Theory motor } \\
\text { speed (rev/min) }\end{array}$ & $\begin{array}{c}\text { Actual motor } \\
\text { speed (rev/min) }\end{array}$ & $\begin{array}{c}\text { Speed difference } \\
\text { (rev/min) }\end{array}$ \\
\hline 1,040 & 200 & 82 & 289 & 295 & 6 \\
\hline 1,240 & & & 338 & 348 & 10 \\
\hline 1,240 & 400 & 84 & 340 & 359 & 19 \\
\hline 1,640 & & & 445 & 459 & 14 \\
\hline 1,240 & 800 & 87 & 338 & 369 & 31 \\
\hline 2,040 & & & 556 & 545 & 11 \\
\hline 1,240 & 1,200 & 88 & 350 & 373 & 23 \\
\hline 2,440 & & & 662 & 648 & 14 \\
\hline
\end{tabular}




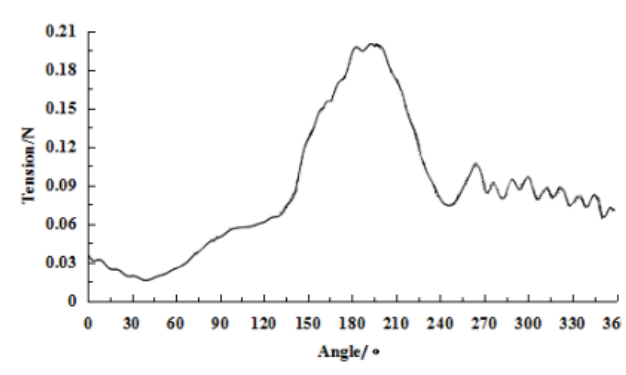

| 1040mm/rack-1240mm/rack

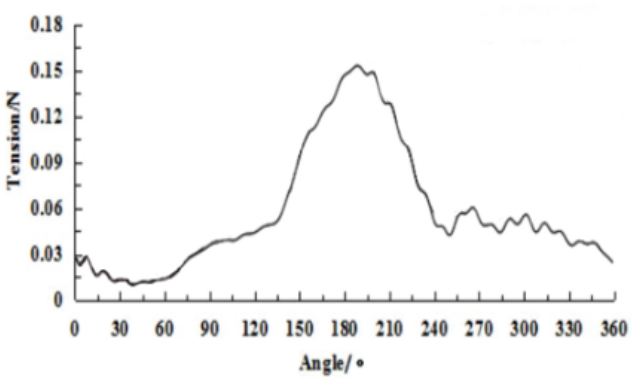

(3)

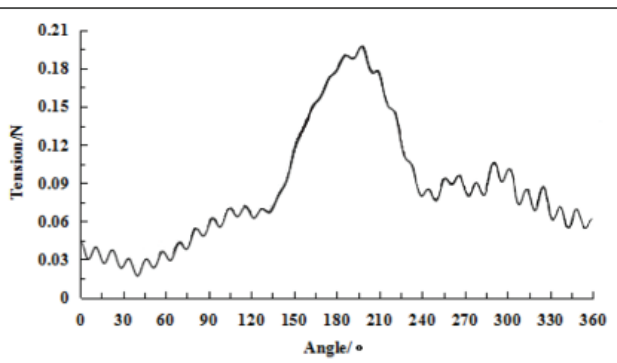

(2)

\section{$1240 \mathrm{~mm} / \mathrm{rack}-1640 \mathrm{~mm} /$ rack}

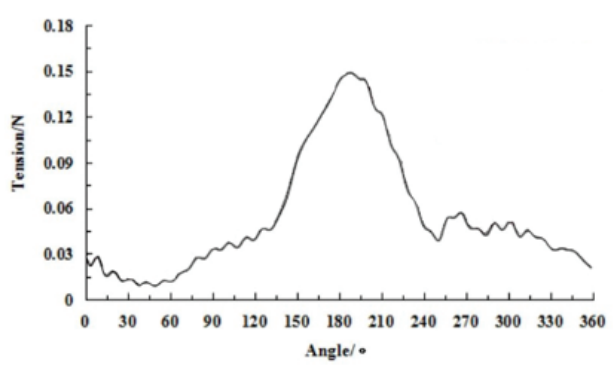

(4)

\section{$1240 \mathrm{~mm} /$ rack-2040mm/rack}

$1240 \mathrm{~mm} / \mathrm{rack}-2440 \mathrm{~mm} / \mathrm{rack}$

Figure 3. The change curve of warp tension with varied let-off amounts switching.

Table 3. Average and maximum tension of warp yarns with switching varied let-off amounts

\begin{tabular}{|c|c|c|c|c|}
\hline $\begin{array}{l}\text { Let-off amounts } \\
\text { (mm/lack) }\end{array}$ & $\begin{array}{c}\text { Difference of let-off amounts }(\mathrm{mm} / \\
\text { lack) }\end{array}$ & Mean tension & Peak tension & Peak angle \\
\hline 1,040 & \multirow{2}{*}{200} & \multirow{2}{*}{0.086} & 0.196 & 195 \\
\hline 1,240 & & & 0.198 & 196 \\
\hline 1,240 & \multirow{2}{*}{400} & \multirow{2}{*}{0.118} & 0.246 & 196 \\
\hline 1,640 & & & 0.252 & 198 \\
\hline 1,240 & \multirow{2}{*}{800} & \multirow{2}{*}{0.071} & 0.159 & 195 \\
\hline 2,040 & & & 0.162 & 197 \\
\hline 1,240 & \multirow{2}{*}{1,200} & \multirow{2}{*}{0.058} & 0.151 & 195 \\
\hline 2,440 & & & 0.158 & 196 \\
\hline
\end{tabular}

between the actual and theoretical speed of the let-off motor corresponding to the let-off amounts in warp-knitting process, with the increase in the deviation value and switching time of the let-off quantity of braided sequence. Through the investigation of the change of warp tension in the switching process, the warp tension is not fixed during the looping process, but it will periodically fluctuate, particularly larger than that under normal operation with the change in the loop formation. The warp tension fluctuates slightly at a peak angle of $196^{\circ}$. Therefore, this research mainly focused on the changes of the four let-off amounts. In future, we will further study the influence of multiple let-off amounts switching on the let-off system at high speed.

\section{Acknowledgments}

The authors acknowledge the financial supports from the National Science Foundation of China (61772238), the Fundamental Research Funds for the Central Universities (JUSRP52013B), and Taishan Industry Leading Talents (tscy20180224). 


\section{References}

[1] Li, X., Jiang, G., Nie, X., Ma, P., Gao, Z. (2015). Knitting technologies and tensile properties of a novel curved flatknitted three-dimensional spacer fabrics. Autex Research Journal, 15(3), 191-197.

[2] Mao, A. Luo, J., Li, Y., Lin, Y., Han, Y. (2016). Knitted fabrics design and manufacture: A novel CAD system for qualifying bagging performance based on geometricmechanical models. Computer-Aided Design, s 75-76: 6175.

[3] Michalak, A., Kuchar, M., Mikołajczyk, Z. (2017). Dynamic analysis of a warp-knitting machine with pneumatic drive for producing $3 D$ knitted fabrics. Indian Journal of Fibre \& Textile Research (IJFTR), 42(4), 502-505.

[4] Hu, J., Jiang, Y. (2002). Modeling formability of multiaxial warp knitted fabrics on a hemisphere. Composites Part $A$ Applied Science \& Manufacturing, 33(5), 725-734.

[5] Jiang, Y., Chen, X. (2005). Geometric and algebraic algorithms for modelling yarn in woven fabrics. Journal of the Textile Institute Proceedings \& Abstracts, 96(4), 237245.

[6] Wang, Z., Hu, H. (2014). 3D auxetic warp-knitted spacer fabrics. Physica Status Solidi B-Basic Solid State Physics, 251(2), 281-288.

[7] Dufour, C., Boussu, F., Wang, P., Soulat, D. (2017). Local strain measurements of yarns inside of $3 D$ warp interlock fabric during forming process. International Journal of Material Forming, 2017, 1-14.

[8] Zhang, J., Jiang, G., Cong, H., Ma, P. (2016). Relationships between warp-knitted run-in value and process parameters. Journal of the Textile Institute Proceedings \& Abstracts, 108(7), 1157-1163.

[9] Blaga, M., Seghedin, N.-E., Ciobanu, A. R. (2013). Warp knitted fabrics behaviour under dynamic testing. Industria Textila, 64(6), 334-341.

[10] Chen, Q., Miao, X., Mao, H., Ma, P., Jiang, G. (2016). The comfort properties of two differential-shrinkage polyester warp knitted fabrics. Autex Research Journal, 16(2), 9099.

[11] Arumugam, V., Mishra, R., Militky, J., Tunak, M. (2016). Inplane shear behavior of $3 D$ spacer knitted fabrics. Journal of Industrial Textiles, 46(3), 868-886.
[12] Xiong, Y., Miao, X., Zhang, A., Jiang, G. (2016). Computer simulation for warp-knitted brushed fabric with patterned piles. Textile Research Journal, 86(15), 1659-1667.

[13] Janouchova, K., Heller, L., Vysanska, M. (2012). Functional warp-knitted fabrics with integrated superelastic NiTi filaments. Autex Research Journal, 12(2), 34-39.

[14] Carpus, E., Scarlat, R., Bonfert, D., Ene, A., Mihai, C., et al. (2014). Investigation of two-layer knitted structures with conductive fibres content. Industria Textila, 65(3), 145-152.

[15] Pohlen, V., Schnabel, A., Neumann, F., Gries, T. (2012). Optimisation of the warp yarn tension on a warp knitting machine. Autex Research Journal, 12(2), 29-33.

[16] Lee, C. K. H., Ho, G. T. S., Choy, K. L., Pang, G. K. H. (2014). A RFID-based recursive process mining system for quality assurance in the garment industry. International Journal of Production Research, 52(14), 4216-4238.

[17] Ghosh, A., Mal, P., Majumdar, A., Banerjee, D. (2016). Optimization of knitted fabric comfort and UV protection using desirability function. Journal of Engineered Fibers and Fabrics, 11(4), 20-28.

[18] Bivainyt, A., Mikučionien, D., Kerpauskas, P. (2012). Investigation on thermal properties of double-layered weft knitted fabrics. Materials Science, 18(2), 167-171.

[19] Tomoko, Y., Masaru, M. (2009). Clothing pressure of knitted fabrics estimated in relation to tensile load under extension and recovery processes by simultaneous measurements. Textile Research Journal, 79(11), 1021-1033.

[20] Rajan, T. P., Souza, L. D., Ramakrishnan, G., Kandhavadivu, $P$., Vigneswaran, C. (2016). Influence of porosity on water vapor permeability behavior of warp knitted polyester spacer fabrics. Journal of Industrial Textiles, 45(5), 796812.

[21] Ma, P., Chang, Y., Jiang, G. (2016). Design and fabrication of auxetic warp-knitted structures with a rotational hexagonal loop. Textile Research Journal, 86(20), 21512157.

[22] Renkens, W., Kyosev, Y. (2016). About the automated pattern creation of $3 D$ jacquard double needle bed warp knitted structures. IOP Conference Series: Materials Science and Engineering, 141, 012010. 